\section{MedienPädagogik}

www.medienpaed.com
Zeitschrift für

Theorie und Praxis

der Medienbildung

ISSN 1424-3636

Themenheft Nr. 17: Medien und soziokulturelle Unterschiede

\title{
Die Wissensgesellschaft und die Bildung des Subjekts - ein Widerspruch?
}

\author{
Sebastian Hacke und Stefan Welling
}

\begin{abstract}
Der Beitrag untersucht, inwiefern sich medienpädagogisch relevante Diskrepanzen zwischen dem Diskurs der Wissensgesellschaft und jugendlichem Medienhandeln nachzeichnen lassen. Dazu werden zunächst einige Aspekte des Konstrukts Wissensgesellschaft skizziert, das in seiner gegenwärtig populären Form ins Fahrwasser an volkswirtschaftlichen Verwertungskalkülen orientierter Denkformen geraten ist. Dies wird in Bezug auf die sich daran gekoppelte Programmatik von Kompetenz diskutiert, welche den «homo oeconomicus» beschwört und sich darin konträr zu klassisch bildungstheoretischen Kategorien verhält. Weitergeführt werden diese Überlegungen anhand der Problematisierung des Medienkompetenztheorems, das sich bis heute damit schwer tut, sich von der Vereinnahmung durch zweckrationale und restringierende Tendenzen zu befreien. Argumentiert wird, dass mit Medienkompetenz zu wenig einer milieuspezifischen Differenzierung Rechnung getragen werden kann, die eine wichtige Prämisse für eine soziale Ungleichheiten ernst nehmende Medienpädagogik ist. Als eine Alternative diskutieren wir, inwieweit sich hier praxeologische Perspektiven zum Verständnis jugendlichen Handelns anbieten. Der Beitrag mündet in einige medienpädagogisch fruchtbare Ideen, in deren Zentrum eine reflexivere, stärker am Habitus von Jugendlichen orientierte Praxis steht.
\end{abstract}

\section{$1 \quad$ Einleitung}

Digitale Medien durchdringen heute die meisten Lebensbereiche und viele Handlungsaspekte sind ohne Medienbezug kaum mehr denkbar. Diese anhaltende Mediatisierung (vgl. Krotz 2007) führt dazu, dass immer mehr Menschen, allen voran Jugendliche, ihr soziales und kommunikatives Handeln immer häufiger und differenzierter auf verschiedenste digitale Medien beziehen. Dabei spielt auch Wissen eine zentrale Rolle. Es wird einerseits benötigt, um Medien zu rezipieren, zu distribuieren und zu produzieren. Andererseits dienen die digitalen Medien dazu, beliebiges Wissen zu erwerben. Ihren Widerhall findet die zentrale gesellschaftliche Relevanz von Wissen vor allem im Postulat der so genannten Wissensgesellschaft. Diese dient regelmässig auch als Hintergrundfolie für die Auseinandersetzung mit Fragen der Medienaneignung. Innerhalb der Medienpädagogik spiegelt sich diese Entwicklung insbesondere im Konzept der Medienkompetenz, das ab Mitte der 1990er-Jahre zu ihrer primären Zielkategorie wurde. 
Übersehen wird jedoch häufig, dass verschiedene Lesarten der Wissensgesellschaft nicht gleichberechtigt nebeneinander stehen. So treten z. B. subjektorientierte Ansätze, die vor allem die Bildungsrelevanz der Medienaneignung vor dem Hintergrund der Veränderung von Selbst- und Weltreferenzen thematisieren (vgl. Marotzki/Nohl 2004; Pietrass 2005) weit hinter einen gesellschaftlich dominierenden Kanon zurück, der Wissensgesellschaft primär auf der Grundlage zu erwerbender Kompetenzen buchstabiert, von denen angenommen wird, dass sie für das Leben in der Wissensgesellschaft unverzichtbar sind (vgl. de Haan/Poltermann 2002; Glotz 1999). Die Konsequenzen dieses öffentlichen Diskurses der Wissensgesellschaft bezüglich der Idee der Bildung gehören aber bislang nicht zum Kernbestand medienpädagogischer Reflexionen. Dies verwundert insofern, da sich vor dem Hintergrund der Konzeption der Wissensgesellschaft eine auf funktionale Erfordernisse abgestimmte Perspektive auf Bildung und Kompetenz etabliert hat, die Gefahr läuft, das subjektiv Eigenlogische im Prozess jugendlicher Medienaneignung aus dem Blick zu verlieren (vgl. Thole 2003; May 2004). Verobjektiviert und zum Massstab von Bildungsprozessen erhoben werden vielmehr nur bestimmte, verwertbare Fähigkeiten. Damit wird einer Sichtweise auf Medienkompetenz Vorschub geleistet, die lediglich Aspekte instrumentellen Handelns, Beherrschens und Könnens fokussiert. Milieuspezifische Aneignungsstile geraten dabei ebenso aus dem Blickfeld wie Ideen zur Medienbildung, die gerade das körperlich-sinnliche, nicht zweckrationale jugendlicher Mediennutzung betonen. Insofern stehen dominante, bildungspolitisch populäre Programmatiken der Wissensgesellschaft und milieu- und habitustheoretische Überlegungen bezüglich der Medienaneignung in einem spannungsreichen Verhältnis. Dessen Beachtung erscheint jedoch gerade für gelingende Passungsverhältnisse von jugendlichen Lebenswelten und pädagogischen Angeboten zentral.

\section{Die Wissensgesellschaft und das Primat der Ökonomie}

Die massenhafte Ausbreitung neuer Technologien hat in den Sozialwissenschaften seit etwa Mitte des 20. Jahrhunderts zu veränderten Beschreibungen gesellschaftlicher Realität geführt. Knapp zusammengefasst erscheint die entsprechende Diskussion als ein Versuch, die gesellschaftliche Entwicklung unter den Vorzeichen technologischen Wandels auf einen geeigneten Begriff zu bringen: Es wurden fundamentale Veränderungen von einer «industriellen» über eine «Informations-» (vgl. Otto/Sonntag 1985) hin zu einer «Wissensgesellschaft» (vgl. Hubig 2000) konstatiert. Als ein deutendes Modell (vgl. Immerfall 1998: 254) hat sich die Wissensgesellschaft gegenüber anderen Beschreibungen erfolgreich behauptet und sich als «gegenwärtig populärste Zeitdiagnose» (Bittlingmayer 2001: 15) gegenüber anderen Etiketten durchgesetzt - gleichwohl hat bisher keine paradigmatische 
Entwicklung stattgefunden, die eine präzise Umgrenzung und inhaltliche Bestimmung zulässt (vgl. Maasen 1999; Rammert 2000; Kübler 2005).

Als zentraler Wegbereiter in die Wissensgesellschaft gilt der Entwurf Daniel Bells (1975), den gesellschaftlichen Wandel hinsichtlich bestimmter Dimensionen zu ordnen und zu typisieren, um Prozesse makroökonomischer Veränderungen, der Verschiebungen in Beschäftigungsverhältnissen und des sozialräumlichen Wandels darzustellen. Bell beschreibt das Szenario der Ablösung der Industrie- durch eine nach- bzw. postindustrielle Gesellschaft, in welcher nicht mehr Rohstoffe oder Energie, sondern Gewinnung und Verwertung von Informationen die Grundlage der wichtigsten Produktionsmechanismen darstellen. Theoretisches wissenschaftliches Wissen wird (als axiales Prinzip) zur Quelle und zum Ausgangspunkt gesellschaftlicher Steuerung. Begründet sieht Bell diese Prozesse in technischen Innovationen, insbesondere im Bereich der Mikroelektronik. Durch sie würde eine quantitative und qualitative Vergrösserung von Wissensbeständen ausgelöst, die ihrerseits wiederum auf das Engste mit technologisch-ökonomischem Wachstum verknüpft ist. Auf diese Weise wird der Computer zum wichtigsten materiellen Träger der Entwicklung, etwa indem computergestützte Informationsverarbeitung in Form einer «intellektuellen Technologie» (Bell 1975: 45) zur Voraussetzung von Entscheidungsfindungen bei komplexen Problemen wird. Trotz seiner heute zurecht in Frage gestellten Tragfähigkeit (vgl. Löffelholz/Altmeppen 1994; Steinbicker 2001) liegen in Bells Gesellschaftsentwurf wichtige Grundlagen für den Diskurs der Wissensgesellschaft: Seit den von ihm beobachteten Modernisierungsprozessen ist das mit neuen Technologien in Zusammenhang stehende Wissen in den Mittelpunkt des Interesses gerückt, da es vermittels Technologie expandiert und so als eine strategische Ressource und als Quelle von Mehrwert erscheint.

Die Frage, inwiefern sich an den Gebrauch von Medientechnologien und die Verfügung über das entsprechende Wissen bestimmte Optionen knüpfen steht im Mittelpunkt der breit rezipierten Theorie von Stehr (1994; 2001). Dieser geht davon aus, dass sich die gegenwärtige Gesellschaftsform «von dem, was uns bisher (...) vertraut war, radikal unterscheidet» (1994: 24). Interessant ist für ihn daran vor allem die dynamische Ausweitung sozialer Handlungsmöglichkeiten, die in eine durch neue Technologien völlig neu ausgerichtete Bestimmung sozialen Lebens eingebettet ist. Diese sich abzeichnende Wissensgesellschaft repräsentiert für Stehr eine "soziale und ökonomische Welt, in der Ereignisse oder Entwicklungen zunehmend 'gemacht) werden, die zuvor einfach «stattfanden»» (2001: 10). Das heisst: Soziale Veränderungen sind das Ergebnis eines Handlungskalküls und lassen sich Wissen als dem zentralen Bestandteil von Handlungskapazität zuschreiben. Deshalb hält es Stehr nur dann für berechtigt, von Wissen zu sprechen, wenn diesem eine «aktive Funktion im gesellschaftlichen Handlungsablauf» (ebd.: 8) zukommt und es dazu befähigt, "etwas in Gang zu setzen» (ebd.: 10) - mit anderen Worten, es sich als strategisch erfolgreich erweist. Mit dieser Charakterisierung 
von Wissen als Zentralbedingung für die Teilhabe an der Herstellung sozialer Tatbestände konzeptualisiert Stehr Wissen als eine Kategorie mit starker Bindung an eine instrumentelle Funktionalität. Es wird rationalisiert und mit objektivierten Wirkungsgraden ausgestattet. Wissen, so die Schlussfolgerung, fokussiert in seiner entscheidenden Relevanz vor allem "ein Wirtschaftssystem», in welchem es «zur wichtigsten Quelle des Wertzuwachses wird» und dadurch eine «erhöhte Produktion unter geringerem Einsatz von Arbeit» möglich wird (Stehr 1994: 524), kurz: Wissen ermächtigt das Subjekt, sich im Kontext von Produktionsprozessen und -beziehungen flexibel anzupassen.

Auf dieser Grundlage hat sich im Diskurs der Wissensgesellschaft ein Wissensbegriff konstituiert, dessen Bedeutung vorrangig bezüglich einer Wertschöpfungskette gesehen wird und auf den - unter Rekurs auf Stehr - ausdrücklich auch in (bildungs-)politischer Programmatik Bezug genommen wird (vgl. etwa den Bericht der Enquete-Kommission des Deutschen Bundestages 2002). Wissen wird als «Rohstoff und Produktionsfaktor» (Josczok 2001: 33) verstanden, dessen aktuelle und zukünftige Bedeutung vor allem im Bereich wirtschaftlich-technischer Leistungsfähigkeit zu sehen sei. In diesem Kontext würden digitale Technologien zum wichtigsten Faktor volkswirtschaftlicher Prosperität: So heisst es im Aktionsprogramm der Bundesregierung zur Informationsgesellschaft 2006, Computertechnik sei für Deutschlands Zukunft der Schlüssel, «um Wissen und Innovation zur Steigerung von Wachstum, Wettbewerbsfähigkeit und Beschäftigung im Zeitalter einer vernetzten Welt zu nutzen» (Bundesregierung 2003: 5). Wissen sei «die Basis für die Innovations- und Wettbewerbsfähigkeit der Wirtschaft» (ebd.: 23) und der Einsatz von Informations- und Kommunikationstechnologie (luK) «spielt dabei eine zentrale Rolle», denn nur die Volkswirtschaften, in denen «Entwicklung und Einsatz leistungsfähiger luK vorangetrieben wird und die in das Wissen des Einzelnen investieren, werden den Übergang in die (...) Wissensgesellschaft erfolgreich bestehen können» (ebd.). Weil im Prozess zunehmender Globalisierung wissensbasierte Innovationen die wirtschaftliche Entwicklung dominierten, werde das Wissen «ökonomisiert und damit wird der Umgang mit Wissen zu einer zentralen Frage der künftigen Gesellschaft» (ebd.). Auch aus Sicht der OECD geht es vor allem darum, «Humankapital (...) als Schlüsselfaktor für das Wirtschaftswachstum und die Verbesserung des wirtschaftlichen Erfolges des Einzelnen» zu fördern (OECD 2005: 11). Schon diese kurzen Skizzen verdeutlichen, dass der gesellschaftlich-politisch dominante Diskurs der Wissensgesellschaft eine soziale Realität konstruiert, in der nur demjenigen "Definitionsmacht im System» zugestanden wird, der «Wissen» im Produktionsprozess gezielt und rasch einzusetzen vermag» (Stross 2001: 208). Dies geschieht unter der Prämisse, die Wissensgesellschaft als Prozess gesellschaftlicher Neuerungen von ökonomischem Format zu deuten, denen sich das Subjekt einerseits nicht entziehen kann und die andererseits, gleichsam automatisch, dazu führen, dass ein Grossteil der Subjekte nicht mehr in der Lage ist, 
daran teilhaben zu können (vgl. Glotz 1999, 2004). Somit erscheint der dominante Diskurs der Wissensgesellschaft als eine Beschreibungsfigur interessengeleiteter Transformationen, aus denen heraus das mit digitalen Technologien in Verbindung stehende Wissen in einer Dimension von Leistung, Effektivität und Wettbewerbsfähigkeit interpretiert wird.

In diesem Kontext lässt sich die klare Tendenz einer einseitigen Verkürzung des Bildungsbegriffs feststellen, die auf einem individualbezogenen, an ökonomischen und zweckrationalen Zielen ausgerichteten, soziale Ungleichheit nicht berücksichtigenden Erwerb von Wissen als zentralem Modus von Bildung gründet und die öffentliche Diskussion über die Ausrichtung von Bildung über weite Strecken prägt. Analog dazu erfolgt die Bewertung von Bildungsinstitutionen und deren Legitimität zunehmend unter Effektivitäts- (d. h. unter dem Aspekt ihrer Zielbezogenheit) und Effizienzkriterien (d.h. unter der Zielstellung einer möglichst optimalen Input-Output-Relation) (vgl. Weber 2002; Wimmer 2002; Cleppien/Kutscher 2004).

\section{Kompetenz in der Wissensgesellschaft}

In der öffentlich dominanten Form der Wissensgesellschaft ist der Umgang mit Computer und Internet zur Idealvorstellung avanciert, sich, ausgestattet mit den nötigen technologischen Fähigkeiten, erfolgreich in einer digitalisierten Welt zu bewegen und zu bewähren. Der Umgang mit Medientechnologien und ein entsprechendes Wissen wird für Heranwachsende zum zentralen Handlungsmodus "to function comfortably as a productive citizen in a computer-oriented society", wie es Watt bereits 1980 formuliert hatte (ebd.: 3). Verbunden ist darin die Vorstellung sozialen Handelns mit einer gesellschaftlich-technischen Rationalität und das Postulat der in diesem Rahmen gesteigerten Handlungspotenziale. Zur Beschreibung der Fähigkeit, die Optionen der Wissensgesellschaft ausschöpfen zu können, hat sich seit den 1990er-Jahren das Konstrukt der Kompetenz etabliert (vgl. Höhne 2003).

Mit der ihr eigenen Fassung von Kompetenz transportiert die Wissensgesellschaft damit eine pädagogische Zielvorstellung, die bestimmte Geltungsansprüche impliziert und begründet. Nimmt man die Forderung Klafkis ernst, Zielvorstellungen aus Sicht der Erziehungswissenschaft in «kritisch-analytischer Art» daraufhin zu überprüfen, «ob sich in ihnen unreflektierte gesellschaftliche Interessen und Machtpositionen ausdrücken», indem man fragt, inwiefern davon die "gegenwärtigen, selbst wiederum historischen Lebensbedingungen des Individuums» tangiert werden (Klafki 1996: 161), offenbart sich, wie folgenschwer die stillschweigenden Implikationen der Wissensgesellschaft für das Subjekt sind. Dies gilt besonders für den Zuwachs an gesellschaftlichen Handlungsoptionen, den Stehr (2001: 13) euphorisch - und darin ebenso einseitig wie affirmativ - als «befreiendes Handlungspotenzial» von Wissen gedeutet hatte. So ist etwa mit Beck (2005) daran zu erinnern, dass zum einen Gestaltungspotentiale und Unsicherheiten gleichzeitig 
wachsen und dass zum anderen aus der Ambivalenz von Kompetenz- und Flexibilitätszumutungen eine Semantik der erhöhten Eigenverantwortung des Einzelnen, seiner Mobilität und seiner Flexibilität folgt, was sich in einer Hineinverlagerung der permanenten Bewältigungen neuer Anforderungen in die individuelle Verantwortung, das Selbstmanagement der Subjekte manifestiert.

Welche Konsequenzen dieser Prozess für den Kompetenzbegriff hat, zeigt etwa Höhne (2007): Er verdeutlicht, dass die Wissensgesellschaft «das zentrale soziologische Referenzkonzept für den Kompetenzdiskurs darstellt und noch schärfer formuliert: Dass die Popularität des Kompetenzbegriffs nicht ohne den wissensgesellschaftlichen Diskursrahmen denkbar ist» (ebd.: 34). Vor allem findet innerhalb dieses Diskursrahmens eine «Anthropologisierung des Kompetenzbegriffs» statt, in dem sich an die konstatierte Zunahme von Anforderungen an die Akteure eine «Dauerthematisierung des Individuums und seiner vermeintlichen Fähigkeiten» knüpft (ebd.). Diese enge Verbindung der Semantik der Wissensgesellschaft mit dem Kompetenzdiskurs bedeutet für das Subjekt, dass es neben der Permanenz der Selbstrationalisierung, die es in Kategorien von Flexibilität und Unternehmertum anruft, zur Zuschreibung von Fähigkeiten kommt. Hierdurch wird Kompetenz zur Charakterisierungsfigur des Subjekts schlechthin: der Kompetenzdiskurs konstituiert somit einen Raum «möglichen und sozial erwünschten Handelns» und wirkt wie eine Sphäre «öffentlicher Beobachtung, der die Haltung dauernder Selbstevaluation der Subjekte hervorbringt» (Höhne 2007: 41). Zugespitzt formuliert bedeutet dies: Wer nicht kompetent ist, gerät unter Verdacht. In einer solchen Wissensgesellschaft werden jugendliche Akteure rechenschaftspflichtig, falls der Umgang mit Computermedien für sie wenig Relevanz hat, oder ihre Computermedienpraxen in Bahnen verlaufen, die sich der gesellschaftlichen Akzeptanz entziehen (vgl. z. B. Pfeiffer et al. 2007). Das Konzept der so genannten digitalen Spaltung steht über weite Strecken synonym für diese Entwicklung, denn darin werden Differenzen im Umgang mit digitalen Medien vorwiegend als Defizite wahrgenommen, was nicht zuletzt dem Design der einschlägigen Untersuchungen geschuldet ist (vgl. zur Kritik Krotz 2007: 286).

Aus der Wissensgesellschaft als einer Beschreibungsfigur, die Heranwachsende in Kategorien von Flexibilität, lebenslangem Lernen, Kompetenz und Wissen anspricht, resultiert so unter der Hand eine Semantik der affirmativen Leistungsorientierung und der Hierarchisierung. Im Sinne einer sozialen Distinktion geht es um die gekonnte Anpassungsbereitschaft und eine letztlich eigenverantwortete Selbstrationalisierung vor dem Hintergrund struktureller Veränderungen, oder anders ausgedrückt: um eine Fähigkeit, sich vor dem Hintergrund der Metapher des Marktes als des allumfassenden Integrationsmodus sozialen Lebens (vgl. Bröckling 2002: 10) auf verschärfende Bedingungen einzustellen. Kompetenz wird synthetisiert zu einem strategischen Können in Bezug auf strukturelle Anforderungen. Es kommt zu einer Biologisierung und Anthropologisierung von Leistungsbereitschaft, sich 
angesichts aktueller und künftig zu erwartender ökonomisch-technischer Veränderungsprozesse funktionsfähig zu halten.

Inwieweit derartige Kompetenzforderungen Realität ein breites Bildungsverständnis prägen, lässt sich etwa im Kontext entsprechender, neoliberal intendierter, Initiativen beobachten. Vor dem Hintergrund der Überzeugung, dass Deutschland als «ein rohstoffarmes Land (...) seine wirtschaftliche Existenz über immaterielle Güter, also Wissen sichern muss» (Kilius/Kluge/Reisch 2002: 11), wird die Wissensgesellschaft zu einer Formel mit pejorativem Beigeschmack, Kompetenz im Rahmen modernisierter gesellschaftlicher Bedingungen zur ideologischen Programmatik: Für Jürgen Kluge, Chef der Unternehmensberatung McKinsey, ist die "gebildete Persönlichkeit» (Kluge 2003: 14) diejenige, die bereitwillig und risikofreudig daran arbeitet, eine individuelle Lebenshaltung einzunehmen, in der sie sich als «Lebensunternehmer» definiert, um im globalen Wettbewerb zu überleben. In z. T. drastischen Formulierungen wird diese Haltung von Kluge direkt mit Fragen der Existenzberechtigung in Verbindung gebracht: Wer in dieser Situation nicht mithalten kann, «wird aus dem Rennen geworfen» (ebd.: 45). Ausschliesslich die mit einem entsprechenden Selbstbewusstsein und entsprechenden Fähigkeiten ausgestatteten Subjekte seien "auf Dauer die einzige Ressource. Alles andere ist Blech, Beton und tote Materie» (ebd.: 48). In einem ähnlichen semantischen Feld positionieren sich auch die Produkte von Technologieherstellern. Nortel verspricht z. B. "Business without boundaries" und Hewlett-Packard bietet «Solutions for the adaptive enterprise". Diese Wissensgesellschaft codiert Kompetenz im Kontext digitaler Medien als Marktvorteil, der dem Subjekt erfolgversprechende Chancen eröffnet. Ausgeblendet bleibt, dass diese Chancen als kulturelles Kapital im Sinne Bourdieus schon immer an eine gesellschaftlich privilegierte Elite adressiert sind.

\section{$4 \quad$ Halbierung der Bildungsidee}

Der Wissensgesellschaft als einer Kategorie von bildungspolitischem Format und ihrer Stilisierung zur zentralen Leitvorstellung steht begründete Kritik in der Erziehungswissenschaft gegenüber. Im Zentrum dieser Kritik steht die Beobachtung, dass mit einer schrittweisen Veränderung von Sichtweisen auf Konzepte von Wissen und Kompetenz eine Funktionalisierung des Bildungsbegriffs legitimiert und Bildung gewissermassen in den Dienst der Wissensgesellschaft gestellt wird. Diese Orientierung bezeichnet Scherr (2002) als Tendenz, die «ökonomischen Erfordernisse der Wissens- und Informationsgesellschaft als ausschliesslichen Massstab zu etablieren», an welchem "Sinn und Legitimität aller pädagogischen Bemühungen bemessen werden» (ebd.: 105). Für die Pädagogik bedeute dies eine Abwendung von «Erfahrungen, Bedürfnissen und Interessen ihrer Adressaten» (ebd.), weil auf diese Weise ignoriert werde, wie die Subjekte «in Auseinandersetzung mit den je eigenen biografischen und aktuellen Erfahrungen zu einem rational begründeten Selbst- und Weltverständnis» gelangen (ebd.: 96). Auf eine damit verbundene 
semantische Verschiebung macht Messner (2003) aufmerksam, in dem er herausarbeitet, wie mit der gegenwärtigen Verschiebung des Fokus von Bildung auf Literacy versucht wird, «eine moderne, für den Übergang von der Industrie- zur 〈Wissensgesellschaft〉 adäquate Bildungskonzeption zu entwickeln» (ebd.: 405), mit der die fortgeschrittenen Industrieländer vor allem auf einen erhöhten Qualifikationsbedarf reagieren. Akzentuiert wird damit eine «sachstrukturell-kognitive Intelligenz (...), wie sie durch die Mathematik und die Naturwissenschaften geleistet wird» (ebd.: 408). Ins Zentrum von Bildung rückt damit allein die «dynamische Rekonstruktion jener Sachgebiete (...), über welche die künftige Wissensgesellschaft expandiert» (ebd.). Noch schärfer liest sich die Kritik von Ruhloff (2006), der die Gefahr sieht, dass vor einem solchen Hintergrund das «Freiheitsversprechen, das mit Bildungsaufgaben verbunden ist, in Konkurrenzvorteilskalkulation» umgeschrieben zu werden droht (ebd.: 35), was einer «Verkehrung des pädagogischen Ermöglichungsgedankens zu einem pädagogisch abwegigen Herstellungsimperativ nach vorgegebenen Leistungsnormen» entspricht (ebd.: 36). Wie auch immer die Kritik akzentuiert wird: Ihr verbindendes Element ist, dass es der öffentliche Diskurs der Wissensgesellschaft erschwert, Bildung nicht allein als die Fähigkeit zur Bewältigung marktförmig strukturierter Ansprüche zu denken, sondern darin die Entwicklung eines subjektiv-sinnhaften Lebensentwurfs mit einzuschliessen, welcher die Gestaltung einer autonomen Lebenspraxis beinhaltet.

Bezüglich des sich hier andeutenden Missverhältnisses zwischen Subjektbildung und Qualifikation ist bildungstheoretisch daran zu erinnern, dass diese auf der Folie der Wissensgesellschaft stattfindende Entwicklung eine Vereinseitigung der Bildungsidee forciert. Bildung, so hatte es Wilhelm Dilthey formuliert, müsse «zwei getrennt auftretende Zielpunkte» in den Blick nehmen: Sie solle «den Individuen eine sie befriedigende wertvolle Entwicklung» und "den Gemeinschaften den höchsten Grad von Leistungskraft verschaffen» (Dilthey 1971: 50). Das sich aus dieser Zweiseitigkeit ergebende Verhältnis formuliert Benner als das Problem einer Vermittlung «zwischen der individuellen und der gesellschaftlichen Bestimmung des Menschen», welche einerseits "die Individuen weder der Gesellschaft opfert», noch andererseits «die menschliche Gesamtpraxis auf ein Spielfeld individueller Willkür und Selbstverwirklichung reduziert» (Benner 1987: 123). Aus einer so beschriebenen Zweiseitigkeit von "gesellschaftlicher Brauchbarkeit» und «individueller Entfaltung» resultierte historisch die Gegenüberstellung von Spezialbildung und allgemeiner Bildung. Dieser bildungstheoretische Blick verdeutlicht, wie stark der Diskurs der Wissensgesellschaft das Verhältnis von Subjektbildung und Qualifikation als eines von zwei grundsätzlich anderen erkenntnisleitenden Interessen und anderen Begründungslogiken geleiteten Ideen verschärft. Grob und Maag Merki (2001) beschreiben dieses spannungsreiche Verhältnis derart, dass die ursprünglich bildungstheoretisch inspirierte Idee der Entfaltung von Dispositionen und Neigungen nicht mehr wie in der Bildungsidee «über das Ziel der individu- 
ellen Selbstentfaltung» begründet wird, sondern vielmehr aus einem «sukzessive wachsenden ökonomischen Qualifikationsbedarf» (ebd.: 49), da die «jeweilige nationale Wirtschaft» durch eine optimale Ausschöpfung der individuellen Potenziale ihre «internationale Wettbewerbsfähigkeit» zu sichern und auszubauen versucht (ebd.).

Deutlich geworden ist, wie unter den Vorzeichen der Wissensgesellschaft über weite Strecken eine Pointierung von Bildung auf operative Fähigkeiten stattfindet, «die im ökonomischen Verwertungskreislauf gebraucht und vermarktet werden können» (Kübler 2005: 138). Inwiefern dies gerade auch für den Umgang mit Medien zutrifft, zeigt sich daran, dass es in Mode gekommen ist, diesen unter bewusstem Verzicht des Bildungsbegriffs als eine Schlüsselqualifikation zu apostrophieren (Baumert 2002: 111). Damit wird medienbezogenes Handeln Jugendlicher ausschliesslich aus der Perspektive der Evaluation aktuellen Vorhandenseins funktionsrelevanter Kenntnisse und Fähigkeiten wahrgenommen, weil es vor allem «zweckorientierten Anforderungen von Qualifikation und gesellschaftlicher Integration» zu genügen habe (Dörpinghaus/Poenitsch/Wigger 2008: 143). Würde sich die Medienpädagogik allein dieser Programmatik verschreiben, hiesse das, von der Idee der Bildung abzurücken. In den Worten Radtkes hätte sie das jugendliche Subjekt zu jenem «homo oeconomicus» zu formen, «dessen rationales Wahlverhalten von der Bildungsökonomie bereits vorausgesetzt wird» (2006: 52), etwa indem sich Medienpädagogik thematisch auf die Beschäftigung mit effizienten Lehr-Lernarrangements und der Vermittlung von Wissen und Kompetenz konzentrierte, die dem Jugendlichen «seine Inanspruchnahme durch die Wirtschaft erleichtern und seine individuelle Karriere sowie sein persönliches Einkommen sichern» (ebd.). Es ginge dann allein darum, Heranwachsende in Form von EDVKursen, Trainingsprogrammen und dem Erwerb von Computerführerscheinen «fit» für die Wissensgesellschaft zu machen. Für alltagsbezogene und biographische (Sinn-)Orientierungen individueller Medienpraxen brauchte man sich, wenn überhaupt, nur insofern zu interessieren, ob sie volkswirtschaftlich nützlich sind - oder eben nicht. Unberücksichtigt bleibt dabei, dass jugendliche Praxen eingebettet sind in sinnhafte Orientierungen, die kollektiven und darin auch milieuspezifisch gefärbten Relevanzen und biographischen Entwürfen viel eher folgen als es das Konzept vom homo oeconomicus zu fassen vermag. Damit soll allerdings, um es ganz deutlich zu sagen, gerade nicht einer jugendlichen Autonomie das Wort geredet werden, ganz so, als ob ihr allein, z. B. in Form jugendkulturell konnotierter Medienpraxen, bildende Momente zukommen. Im Gegenteil ist die Abarbeitung an gesellschaftlich strukturierten Anforderungen ein zentraler Bildungsaspekt, der jedoch in seiner Wahrnehmung an milieuspezifische Formationen des Habitus gebunden ist, die es in pädagogischer Absicht gilt zu rekonstruieren und angemessen zu begleiten, anstatt sie lediglich zu affirmieren. Eine Halbierung der Bildungsidee scheint vielmehr dann gegeben, sollten die aus der Alltagspraxis emergierenden 
Medienaneignungsformen Jugendlicher in ihrer Rationalität nicht mehr als konstitutiver Bestandteil eines Bildungsprozesses (an)erkannt werden.

\section{Quo vadis Medienkompetenz?}

Die beschriebenen Herausforderungen und Schwierigkeiten lassen sich anhand des Medienkompetenztheorems weiterführen. Vor allem auf die Arbeit von Dieter Baacke gründend, avancierte das Konzept der Medienkompetenz in den 1990erJahren zur Zieldimension bzw. zum Versuch der pädagogisch-handlungspraktischen Konkretisierung der Medienpädagogik. Dabei zeigte sich jedoch schnell, dass der Sinn, Medienkompetenz zu vermitteln, in nahezu allen institutionalisierten Lernorten restringierte Tendenzen aufwies (vgl. Schell 1999: 391). Sich ebenfalls auf Schell (1999) beziehend stellen Bausch und Sting (2001) fest, dass die dortigen Überlegungen zur Medienkompetenz vor allem auf die Befähigung des Individuums zu einem zweck- und zielgerichteten Mediengebrauch setzen: Die Fähigkeit, Medien «bestimmungsgemäss zu nutzen, wird zu einer Entwicklungs- und Bildungsaufgabe erklärt, die medienbezogenes Handeln auf pädagogisch akzeptierte Praktiken beschränkt» (ebd.: 255). Ergänzend weist Roland Bader darauf hin, dass diese Aufgabe zunehmend unter Qualifizierungsaspekten bearbeitet werde. Die Frage nach Möglichkeiten des sozialen Lernens sowie nach Partizipationsmöglichkeiten, gerät an den Rand der Medienpädagogik bzw. wird dort gar nicht mehr berücksichtigt. Dadurch ist die sozialisatorische Perspektive in den konzeptionellen Entwürfe der Medienpädagogik seit Mitte der 1990er-Jahre zunehmend in den Hintergrund geraten (Bader 2001: 357).

Interessanterweise weist Baacke (1996: 121) selbst auf die Schwierigkeiten hin, die mit der rationalistischen Verengung des Medienkompetenzbegriffs einhergehen. Dabei werde u. a. die Körperlichkeit und die Emotionalität menschlichen Handelns in den meisten Fällen ausgeblendet und Medienkompetenz in ein Zweck-Mittel-Schema eingebunden. Dem Alltagshandeln liegen aber nur sehr eingeschränkt solche Schemata zugrunde. Darüber hinaus, so Sutter und Charlton (2002), führt die kompetenztheoretische Fokussierung auf Resultate individueller Lernprozesse zu einer Vernachlässigung sowohl der sozialen Bedingungen als auch der prozessualen Dynamik von Entwicklungs- und Sozialisationsprozessen (vgl. ebd.: 138). Das Nicht-Rationale und das Körperliche sind fundamentale Dimensionen der sozialen Erfahrung, auch wenn dies häufig in Abrede gestellt wird (vgl. Buckingham 2003: 162). So weist z. B. Niesyto (2004) darauf hin, dass es den Kompetenzen Jugendlicher mit Schreib- und Leseschwächen entgegen käme, ihre visuellen und körpersprachlichen Ausdrucksformen zu fördern und ihnen assoziativ-intuitive Aneignungsformen zu ermöglichen (vgl. ebd.: 45). Des Weiteren begünstigten gerade Möglichkeiten zum lustvollen und spielerischen Umgang mit den Medien - verbunden mit Räumen zum Ausprobieren und Experimentieren ohne pädagogische Vorbelegung - das Entstehen neuer Ideen und ästhetischer Ereignisse, die zuvor 
durch ziel- und zweckorientierte Vorgehensweisen verstellt waren (ebd.: 36 f.; vgl. auch Zacharias 2004: 175). Hier entsteht die Frage, ob dieser Anspruch mit dem Begriff der Medienbildung adäquat erfasst werden kann. Um Medienbildung zu forcieren, so etwa Winfried Marotzki (2004), sei zum einen die Stärkung kritischer Reflexionshorizonte erforderlich. Zum anderen bedürfe es einer stärkeren kulturtheoretischen Dimensionierung, um eine umfassendere Perspektive auf die neuen Informationstechnologien zu gewinnen. Medienbildung, so Marotzki, werde damit insgesamt «das kreative Projekt, Menschen Hilfestellungen zu bieten, die Möglichkeit der neuen virtuellen Welten für sich zu erschliessen, um auf diese Weise die Chance zu erhalten, im neuen kulturellen Raum des Internet sich zu bewegen und ihre Stimme zu erheben» (ebd.: 71).

Für ein generelles, nicht altersdifferenziertes Konzept von Medienbildung ist Marotzkis Verständnis von Medienbildung sicherlich adäquat. Für die medienpädagogische Arbeit mit Jugendlichen greift es zu kurz, da es das Risiko eines ähnlichen tendenziell zweckrationalen Impetus trägt wie die traditionellen Konzepte handlungsorientierter Medienpädagogik. Da sich die lernenden Subjekte, als Ziel medienpädagogischer Bemühungen, diesen Ansätzen essenziell entziehen, ist die starke Fokussierung auf selbige und die Vermittlung individueller Kompetenzen in Frage zu stellen. Erfolgversprechender scheint es, solche Szenarien mediatisierter Kommunikation medienpädagogisch aufzugreifen, in denen zunächst gar keine pädagogischen Intentionen erkennbar sind (vgl. auch Kerres et al. 2003: 90 f). Denn Medienbildung «ist im Gegensatz zur -kompetenz nicht eine ausgebildete Fähigkeit für, sondern Resultat von Mediennutzung, was natürlich die Nutzung positiv im Sinne von Medienkompetenz beeinflusst» (Pietrass 2002: 394, Herv. i. Orig.). In diesem Sinne kann selbst der vermeintlich inkompetente Umgang mit Medien bildend sein, da er Anstösse zu geben vermag, die zu einer Veränderung der Selbstund Weltreferenzen der Jugendlichen führen (ebd.). Inkompetent ist aus dieser Perspektive z. B. das Aufrufen pornografischer Internetseiten oder der illegale Musikdownload. Aus einem solchen Blickwinkel heraus eröffnet sich eine neue, weil prinzipiell offene Herangehensweise an die Medienpraxen Jugendlicher. Im Zentrum der medienpädagogischen Praxis steht nicht mehr die Praxis an sich (z. B. das Chatten oder das Computerspielen), sondern deren Beitrag zur Veränderung der Selbst- und Weltreferenzen. Die Frage nach Medienbildung stellt sich dann dahingehend, so Pietrass (2005: 45), wie das Selbst- und Weltverhältnis der Menschen durch Medien geformt wird. Auch hier wird deutlich, dass Unbestimmtheitsdimensionen und unterschiedliche Formen von Geselligkeit konstitutive Elemente von Medienbildung sind, die sich nicht in Ablaufschemata zwängen und beliebig hervorrufen lassen. Vielmehr erfordern sie situativ angepasstes Handeln professioneller Pädagogen, das sich kaum schematisieren lässt. Dennoch haben sie einen grossen Anteil an der Herstellung von Situationen, die für die Jugendlichen gün- 
stige Voraussetzungen schaffen, um Bildungsprozesse im Sinne der Veränderung von Selbst- und Weltreferenzen zu entwickeln.

\section{Die Initiierung von Bildungsprozessen im Kontext milieuspezifischer Differenz}

Für die Konzeptualisierung medienbezogener Bildungsprozesse liegen im bisher Gesagten wichtige Grundlagen: Anstatt ausschliesslich die Erfüllung bestimmter Standards als Bezugspunkt zu nehmen - etwa zu testen, über welche Computerskills Jugendliche verfügen und die Resultate in der Rationalität der empirischquantitativen Bildungsforschung in verschiedene Kompetenzstufen zu kategorisieren - bietet es sich an, danach zu fragen, inwiefern sich Prozesse der Medienaneignung in bestimmte Orientierungen innerhalb der Adoleszenz kontextualisieren. Insbesondere während der Zeit, die mit dem Auslaufen der Schule mittleren Niveaus (Haupt- und Realschule) eingeleitet wird, wird von Jugendlichen allgemein erwartet, dass sie eine weiterführende bildungs- bzw. berufsbiografische Perspektive entwickeln und umsetzen. Die von Bohnsack (1989: 199ff.) rekonstruierte Entwicklungstypik zeigt, wie Jugendliche mit den an sie gestellten biografischen Anforderungen umgehen. Gerade Jugendliche mit geringer formaler Bildungsbeteiligung entziehen sich dieser Entscheidung häufig. Aufgrund fehlender oder nicht realisierbarer berufs- bzw. bildungsbiografischer Vorstellungen markiert der Versuch, diese Orientierungen umzusetzen, für viele den Beginn einer Ent-Täuschungsphase, die eingebettet ist in vielfältige Sinn- und Orientierungsprobleme. Die Problematisierung der eigenen (berufs-)biografischen Entwicklung in diesem Abschnitt mündet regelmässig nicht in eine Suche nach Handlungsalternativen. Stattdessen schliesst sich ihr eine Phase der Negation an, begleitet von einer krisenhaften suspendierenden Haltung (vgl. ebd.: 202). Ein derartiger Höhepunkt der Adoleszenzkrise scheint gerade für Jugendliche mit niedrigem formalem Bildungshintergrund nichts Besonderes zu sein, unabhängig ob es sich um einheimische (vgl. Bohnsack 1989; Bohnsack u. a. 1995; Schäffer 1996) oder um Jugendliche mit Migrationshintergrund handelt (vgl. Nohl 2001). Bei letzteren umfasst die Negation von Sinnhaftigkeit aber nicht nur Aspekte der Arbeits- und Berufsbiografie, sondern auch der familiären und gesellschaftlichen Beziehungen (vgl. ebd.: 256). Derartige Orientierungs- und Sinnkrisen der Adoleszenz treten dort in verschärfter Form auf, wo «eine Integration in Gemeinsamkeiten und Selbstverständlichkeiten, d. h. in habituelle Übereinstimmungen der milieuspezifischen Alltagspraxis nicht bruchlos gegeben und somit biografische Diskontinuitäten zu beobachten sind, also unter Bedingungen milieuspezifischer Desintegration» (Bohnsack/Nohl 1998: 266). Bei den bildungserfolgreichen Jugendlichen verläuft diese Krise zumeist in abgeschwächter Form. Zum einen stellen sich ihnen (berufs-)biografisch relevante Entscheidungen erst zu Anfang der mittleren Jugendphase im Alter zwischen 17 und 19 Jahren. Zum anderen sind sie darauf besser vorbereitet, da Motive in Form 
vorausschauender theoretischer Entwürfe ihre biografische Selbstverortung bestimmen und die individuelle karriereförmige Entwicklung den übergreifenden Rahmen ihrer biografischen Selbstverortung liefert (vgl. Bohnsack 1989: $217 \mathrm{ff}$.).

Der oben angedeutete Versuch der Jugendlichen, sich aus ihrem Alltag situativ zu befreien und der bis zur vollständigen Negation der (berufs-)biografischen Praxis führen kann, erfolgt über weite Strecken auf der Basis von Aktionismen. Da der Höhepunkt dieser Adoleszenzkrise gemeinhin im Rahmen der Peergroup erlebt wird, sind die Aktionismen unmittelbar an das kollektive Handeln gebunden (vgl. Nohl 2006: 86 f.). Sie konstituieren ein zentrales Strukturmerkmal aktueller Jugendphasen und Jugendkulturen (vgl. Bohnsack/Nohl 2001: 18). Die korrespondierende Handelungspraxis ist auf einer vorreflexiven Ebene angesiedelt, d. h. es liegt ihr kein zweckrationaler Handlungsentwurf und der daraus resultierende Vollzug einer an diesem Entwurf orientierten Handlung zugrunde (vgl. ebd.: 20; Bohnsack 1997). Aktionismen sind stattdessen eine spezifische Form von Ritualen, die nicht auf die Bestätigung und Re-Organisation von kollektiven Wissensbeständen, sondern auf deren Entstehung gerichtet sind. Somit ist auch deren Ausgang weitgehend offen- auch im Hinblick auf die damit verbundenen Chancen und Risiken (vgl. Bohnsack/Nohl 2001: 23). Chancen eröffnen sich insbesondere im Zuge der, im Verlauf der Adoleszenzkrise bei den allermeisten Jugendlichen früher oder später einsetzenden Re-Orientierungs- und Sinnfindungsphase, bei der der Peergroup ebenfalls eine zentrale Bedeutung zukommt. In ihr wird die Suche nach habitueller Übereinstimmung und milieuspezifischer Zugehörigkeit bzw. kollektiven Orientierungen u. a. auf der Grundlage gruppenhafter Aktionismen erprobt (vgl. Bohnsack/ Nohl 1998: 266f.). Vor diesem Hintergrund entwickeln Jugendliche schliesslich biografische Orientierungen, die ihrem Leben (neue) Bedeutungen verleihen und den Ausgangspunkt biografischer Anstrengungen markieren (vgl. Welling 2008: 43ff.). Entscheidend ist, dass sich jugendliche Orientierungsrahmen im Sinne habitualisierter und kollektiver Wissensbestände besonders dort ausbilden, wo sie «nicht nur internalisiert, sondern auch inkorporiert, $d$. h. in den modus operandi der körperlichen und sprachlichen Praktiken eingeschrieben und in diesem Sinne <mimetisch angeeignet werden» (Bohnsack 2003: 132). Das heisst, dass es sich um grösstenteils unbewusste, an Vorbildern und den Handlungen anderer ausgerichtete Prozesse kreativer Nachahmung handelt. Solche mimetischen Prozesse sind, so betonen Bausch/Sting (2001) «sinnlich und beziehen sich daher vornehmlich auf die Ausführung menschlichen Verhaltens». Mit ihrer Hilfe erfolgt die «Herausbildung eines praktischen, handlungsrelevanten Wissens, einer körperlichen Beteiligung und eines leiblichen Habitus» (ebd.: 342). Auch Rituale vollziehen sich handlungspraktisch im Modus des mimetischen Handelns (vgl. Zirfas/Wulf 2001: 195). Da jugendliches Alltagshandeln durch habitualisiertes Handeln in Form von Routinehandlungen ohne bewusste Zweck-Mittel-Verknüpfungen dominiert wird (vgl. Gaffer 2001: 38; Voss 2000: 33), bei dem darüber hinaus die Materialität des 
Körpers zum Tragen kommt (vgl. Gebauer/Wulf 1998: 9), ist es gerade die Infragestellung des körperlich-praktischen Bezugs zur Welt in Form primär unbewusster Gewohnheiten, Routinen und eingespielter Wahrnehmungsweisen - z. B. im Verlauf milieuspezifischer Desintegrationsprozesse - die nach kreativer Bearbeitung im Sinne einer Re-Orientierung ruft (vgl. Joas/Knöbl 2004: 712). Es kommt zu Situationen, in denen «die Selbstverständlichkeit der Lebenswelt quasi aufgehoben wird, in denen plötzlich eine andere Wirklichkeit erscheint bzw. die Möglichkeit einer anderen Wirklichkeit gedacht werden kann» (ebd.: 234).

Mit dieser Perspektive verbinden sich für die Medienpädagogik Chancen: Zunächst wird deutlich, dass Jugendliche in jegliche pädagogische Arrangements immer schon grundlegende biografische Orientierungen einbringen bzw. auf der Suche nach neuen Orientierungen sind. Daran gilt es in rekonstruktiver Perspektive anzuschliessen, um ihnen als Adressaten pädagogisch und bildungsadäquate Angebote machen zu können. Gleichzeitig würden diese Angebote die Autonomie jugendlicher Subjekte anerkennen und von einer Diskrepanz von pädagogisch intendierten Zielwerten und den eigenen Konstruktionen ihrer Adressaten ausgehen. Damit würde der vordergründigen Semantik des Fit-Machens, die mancherorts den "Charakter eines von den biografischen Orientierungen der Jugendlichen entkoppelten Globalziels angenommen» hat (Welling 2008: 212) von vorneherein eine deutliche Absage erteilt. Erforderlich dazu wäre es, die Auseinandersetzung Jugendlicher mit medienbedingten Optionen nicht allein in Relation zum funktionalen Nutzwert für ein Leben in der Wissensgesellschaft zu denken, sondern zu allererst als einen mit ihrer Lebenspraxis vermittelten, subjektiven Bildungsprozess.

\section{Konsequenzen und medienpädagogische Anschlussmöglichkeiten}

Ob für die medienpädagogische Praxis als Leitbegriff nun Medienkompetenz oder Medienbildung vorzuziehen ist, soll hier nicht entschieden werden. Zumindest erscheint bezüglich des Gegenstands der Medienbildung erheblicher Bedarf nach einer Schärfung des Begriffs, um inn im angesprochenen Sinne zu operationalisieren und nutzbar machen zu können. Die Literaturlage zur Medienbildung ist jedoch uneinheitlich, bisweilen widersprüchlich (vgl. z. B. Herzig 2001; Fussmann 2003). In dieser Situation könnte Baackes Hinweis auf die Berücksichtigung einer Unterhaltungskompetenz weiterhelfen (vgl. 1996: 121), welche von Vorschlägen zu einer affektiven Dimension aufgegriffen worden ist, die Emotionen als wichtige Aspekte der Mediennutzung bezeichnen (vgl. Aufenanger 2001: 119f.). In die gleiche Richtung zeigt auch die Adressierung einer Dimension medienbezogener Genussfähigkeit (vgl. Groeben 2002: 164), die ausserdem unterstreicht, das Vergnügen und Spiel bzw. das Ludische zentrale Aspekte im Umgang mit Medien sind (vgl. Buckingham 2003: 162). 
Gleiches gilt für die ästhetische Dimension, die den Medien laut Zacharias (2004) entsprechend ihrer gestalteten Formen und deren sinnlicher Wahrnehmbarkeit immer immanent sei. «lm «Ästhetischen` fallen Sinn und Sinnlichkeit zusammen, ästhetische Erfahrung, Rationalität, Erkenntnis und Aktivität haben eigenwertigen Charakter, mediale Qualitäten und schliessen Emotionen bewusst und wirkungsvoll ein: Das ist das besondere Vermögen des Sinnlich-Ästhetischen in der Differenz zum Rational-Abstrakten, zum Reflexiv-Theoretischen» (ebd.: 176). Mittels einer stärkeren Fokussierung ästhetischer Aspekte können darüber hinaus verhärtete Strukturen individueller Wahrnehmung und sozialer Verständigung aufgebrochen und körperliche und nicht rationale Aspekte des Handelns verstärkt zugelassen werden, um so die Individuen für ihre eigenen Wünsche und Belange und völlig Neues zu sensibilisieren (vgl. Ehrenspeck/Rustemeyer 1996: 383). Hörster und Müller (1996: 623) zufolge sind es gerade solche Tätigkeiten und Fähigkeiten, die sich rationalem Kalkül und Verstehen über weite Strecken entziehen und am Anfang (medien)pädagogischen Handelns stehen können. Sofern sie Verständigung ermöglichen, stellen sie die Grundlage für rationales Handeln und Verstehen überhaupt erst her. Mit einer stärkeren Fokussierung einer so verstandenen Medienbildung könnte der Feststellung Rechnung getragen werden, "dass die Unverfügbarkeit des Subjekts sich nach seinen eigenen generativen Ausdrucksmustern entfaltet, ohne durchweg immer pädagogisch und im pädagogischen Raum angeleitet sein zu müssen» (Baacke 1996: 121).

In dieser Situation erscheint es uns zielführend, wenn medienpädagogischer Bildungspraxis von einer intensiveren Auseinandersetzung mit dem «jugendlichen Habitus» begleitet würde. In diese Richtung weisen auch Diskussionen der neueren Jugendforschung. So argumentiert etwa Paus-Hasebrink (2005: 76 ff.), dass es wenig Sinn mache, an einer Ausgangsperspektive festzuhalten, die von einer Dichotomie zwischen einer äusseren Realität einerseits und einer inneren Realität andererseits ausgeht (Konzepte von Patchwork-Identität, Freiheitsoptionen und Wahlnotwendigkeiten eingeschlossen). Übertragen auf den Medienkontext hiesse dies, die traditionelle Wahrnehmung im Sinne des Schemas «hier das Medium dort der Jugendliche» aufzubrechen und stattdessen viel eher jugendliche Orientierungsmuster in den Blick zu nehmen, und zwar weder nur aus einer subjektiven noch einer nur objektiven Perspektive. Stattdessen ginge es um die Überbrückung beider und eine Fokussierung auf "das je individuelle, aber dennoch über die subjektive Repräsentation hinausweisende Lebensumfeld, das soziale Milieu, also die sozialen Räume, die dem einzelnen tatsächlich oder symbolisch zur Verfügung stehen» (ebd.: 80). Im Zentrum dieser Überlegungen stehen für Paus-Hasebrink die Suche und das Freilegen des praktischen Sinns, verstanden als die unterschiedlichen Modi einzelner Jugendlicher oder einzelner Gruppen bei der Ausbildung von Identität und dem Erwerb von Handlungsmöglichkeiten. Ein solcher Blick erlaubt die Frage nach der Ausprägung des Habitus jugendlicher Akteure, verstan- 
den im Sinne Bourdieus $(1992,1998)$ als eine Art implizite Grammatik des Handelns bzw. Erzeugungs- und Strukturierungsprinzip der unterschiedlichen (Medien-)Praxen. Der Habitus verweist auf den praktischen Sinn und darin sedimentiert sich die Praxis der Vergangenheit, welche sowohl die gegenwärtige als auch die zukünftige Praxis weitgehend autonom anleitet (vgl. Hörning 2001: 167). Um dem gerecht zu werden, müssen für Paus-Hasebrink (vgl. 2005: 80 f.) bei einer Betrachtung Jugendlicher (Medien-)Praktiken folgende drei Bereiche zusammengezogen und miteinander verbunden werden: Handlungsoptionen als gesellschaftlich-strukturell bedingte Voraussetzungen des Handelns, Handlungsentwürfe als subjektive Wahrnehmungen dieser Struktur im Zusammenspiel der Verzahnung von Praxis und handlungsleitender Anschauung, verbunden mit dem jeweiligen sich aus diesem Zusammenspiel entwickelnden Eigensinn sowie entsprechende Handlungskompetenzen, verstanden als die subjektiven Ausprägungen dieses Konglomerats auf der Handlungsebene, die zu einer sinnvollen Alltagsgestaltung eingesetzt werden. Entscheidend ist dabei, Medienaneignung als soziale Praxis zu begreifen und anzuerkennen, dass sich diese durch ein soziales Zusammenleben etabliert, in welchem Erfahrungen, Fertigkeiten und Wissen zwischen den Akteuren ausgetauscht und kultiviert werden (vgl. Grundmann 2006: 29).

Dass ein auf diesem Wege geleisteter Anschluss an jugendliche Orientierungen höchst bedeutsam in Bezug auf gelingende Passungsverhältnisse von jugendlichen Medien- und Lebenswelten und einer pädagogisch-institutionellen Rationalität ist, wie sie sich etwa in schulischen und ausserschulischen Kontexten manifestiert, liegt auf der Hand. Denn bevor der Heranwachsende in diese Zusammenhänge eintritt, hat er bereits entscheidende Prozesse der Formation seines Habitus vollzogen. In Welling (2008) wird exemplarisch gezeigt, wie sich die von uns in Kapitel 3 und 4 kritisierte, stark rationalistische und technisch-ökonomistische Färbung des Kompetenz- und Wissensbegriff auf die der medienpädagogischen Praxis zugrunde liegenden berufsbiografischen Orientierungen auswirkt und welche Schwierigkeiten daraus für die pädagogische Praxis resultieren. Inwiefern eine Passung zwischen jugendlichem Habitus und pädagogischem Handeln misslingt, da sich Beziehung zu den Jugendlichen gerade nicht auf eine Sensibilität für deren individuierte Fallstruktur - also entlang der dem Medienhandeln zugrunde liegenden Orientierung - gründet, zeigt auch ein Fallbeispiel in Hummrich et al. (2006: 33 ff.): Hier geht es um einen Jugendlichen, der in seinen Individuationsbestrebungen mit der Problematik fehlender Anerkennung konfrontiert ist und diese Herausforderung fast ausschliesslich mit dem Spielen von Cyberpunk-Rollenspielen im Internet bearbeitet. In seiner reformorientierten Schule, die sich überwiegend an ökologisch ausgerichteten Milieus orientiert und die eine kritische Haltung gegenüber Medien einnimmt, wird die Identifizierung mit dieser Welt zu einem Problem. Anstatt den Jugendlichen «in seinen Interessen zu würdigen, reagieren die Lehrer mit Abwehr» (Hummrich et al. 2006: 36), da sie dieser Art Medienorientierung keinen prak- 
tischen Sinn zuordnen (können). Versäumt wurde in dem Beispiel demnach, sich hinsichtlich eines sinnvollen Arrangements entsprechender Bildungsangebote a priori der Besonderheit jugendlicher Medianeignung zu vergewissern. Einem solchen Ziel hätte sich eine die soziale Ungleichheit berücksichtigende Medienpädagogik immer wieder neu zu stellen, vor allem auch angesichts der heute zu beobachtenden hochgradig diversifizierten Medienaneignungsformen Jugendlicher. Andernfalls leistete sie einer Form der Wissensgesellschaft Vorschub, in welcher statt der Grundfrage nach Bildung als einer paradoxen Struktur der «Symbiose von funktionalen Erfordernissen und individuellen Entwürfen des Lebens» (Tenorth 1997: 982) nur noch Standortrhetorik und Schlagworte wie «Zukunftsfähigkeit durch IT-Beherrschung» von Belang sind.

\section{Literatur}

Aufenanger, Stefan (2001). «Multimedia und Medienkompetenz - Forderungen an das Bildungssystem». Jahrbuch Medienpädagogik 1. Hrsg. v. Stefan Aufenanger, Renate Schulz-Zander u. Dieter Spanhel. Opladen: Leske + Budrich. S. 109-122.

Baacke, Dieter (1996). «Medienkompetenz - Begrifflichkeit und sozialer Wandel». Medienkompetenz als Schlüsselbegriff. Hrsg. v. Antje von Rein. Bad Heilbrunn: Klinkhardt. S. 112-124.

Bader, Roland (2001). «Legitime Partizipation. Ein Handlungsmodell der Medienpädagogik mit Computern». Bildung in virtuellen Welten. Praxis und Theorie ausserschulischer Bildung mit Internet und Computer. Beiträge zur Medienpädagogik, Band 6. Hrsg. v. Wolfgang Schindler, Roland Bader u. Bernhard Eckmann. Frankfurt/M.: Gemeinschaftswerk der Evangelischen Publizistik, Abt. Verlag. S. 356-376.

Baumert, Jürgen (2002). «Deutschland im internationalen Bildungsvergleich». In: Killius, Nelson et al. (Hrsg.): Die Zukunft der Bildung. Hrsg. v. Nelson Kilius u. a. Frankfurt/M.: Suhrkamp. S. 100-150.

Bausch, Constanze; Sting, Stephan (2001). «Rituelle Medieninszenierungen in Peergroups». Das Soziale als Ritual. Zur performativen Bildung von Gemeinschaften. Hrsg. v. Christoph Wulf u. a. Opladen: Leske + Budrich. S. 249-323.

Beck, Ulrich (2005). «Die Gesellschaft des Weniger. Ein Land steigt ab». Süddeutsche Zeitung, Nr. 27/2005. S. 15.

Bell, Daniel (1975). Die nachindustrielle Gesellschaft. Frankfurt/M.: Campus.

Benner, Dietrich (1987): Allgemeine Pädagogik. Weinheim, München: Juventa.

Bittlingmayer, Uwe H. (2001). «Spätkapitalismus» oder «Wissensgesellschaft??» Aus Politik und Zeitgeschichte. Beilage zur Wochenzeitung «das Parlament», 08/2001, Bd. 36. S. 15-23.

Bohnsack, Ralf (1989). Generation, Milieu und Geschlecht. Ergebnisse aus Gruppendiskussionen mit Jugendlichen. Opladen: Leske + Budrich.

Bohnsack, Ralf (1997). «Orientierungsmuster): Ein Grundbegriff qualitativer Sozialforschung». Methodische Probleme der empirischen Erziehungswissenschaft. Hrsg. v. Folker Schmidt. Hohengehren: Schneider. S. 49-61.

Bohnsack, Ralf (2003). «Orientierungsmuster». Hauptbegriffe Qualitativer Sozialforschung. Hrsg. v. Ralf Bohnsack, Winfried Marotzki u. Michael Meuser. Opladen: Leske + Budrich. S. 132-133. 
Bohnsack, Ralf; Nohl, Arnd-Michael (2001). «Jugendkulturen und Aktionismus. Eine rekonstruktive empirische Analyse am Beispiel des Breakdance». Jahrbuch Jugendforschung Band 1. Hrsg. v. Hans Merkens u. Jürgen Zinnecker. Opladen: Leske + Budrich. S. 1737.

Bourdieu, Pierre (1992). Rede und Antwort. Frankfurt/M.: Suhrkamp.

Bourdieu, Pierre (1998). Praktische Vernunft. Zur Theorie des Handelns. Frankfurt/M.: Suhrkamp.

Bröckling, Ulrich (2002): «Jeder könnte, aber nicht alle können. Konturen des unternehmerischen Selbst» Mittelweg 36, 11. Jg., Heft 4/2002. S. 6-26.

Buckingham, David (2003). Media Education. Literacy, Learning and Contemporary Culture. Cambridge: Polity Press.

Bundesministerium für Wirtschaft und Arbeit und Bundesministerium für Bildung und Forschung (Hrsg.) (2003). Informationsgesellschaft Deutschland 2006. Aktionsprogramm der Bundesregierung. Berlin.

Cleppien, Georg; Kutscher, Nadia (2004). «Digital Inequality und Qualität von Online-Bildung». Informelle Bildung online. Perspektiven für Bildung, Jugendarbeit und Medienpädagogik. Hrsg. v. Hans-Uwe Otto u. Nadia Kutscher. München, Weinheim: Juventa. S. 80-96.

de Haan, Gerhard; Poltermann, Andreas (2002). Funktion und Aufgaben von Bildung und Erziehung in der Wissensgesellschaft. Paper 02-167 der Forschungsgruppe Umweltbildung Berlin: Verein zur Förderung der Ökologie im Bildungsbereich e. V.

Deutscher Bundestag (Hrsg.) (2002). Schlussbericht der Enquete-Kommission «Globalisierung> der Weltwirtschaft. http://www.bundestag.de/gremien/welt/glob_end/index.html (01.02.09).

Dilthey, Wilhelm (1971). Schriften zur Pädagogik. Besorgt von Hans-Hermann Groothoff und Ulrich Herrmann. Paderborn: Schöningh.

Dörpinghaus, Andreas; Poenitsch, Andreas; Wigger, Lothar (2008). Einführung in die Theorie der Bildung. 2., durchges. Aufl., Darmstadt: Wissenschaftliche Buchgesellschaft.

Ehrenspeck, Yvonne; Rustemeyer, Dirk (1996). «Bestimmt unbestimmt». Pädagogische Professionalität. Untersuchungen zum Typus pädagogischen Handelns. Hrsg. v. Arno Combe u. Werner Helsper. Frankfurt/M.: Suhrkamp. S. 368-390.

Fussmann, Albert (2003). "Medienbildung in der Jugendarbeit - Mehr Bildung in der Jugendarbeit». Medienbildung. Beiträge aus Theorie und Praxis von Schule und Jugendarbeit. Hrsg. v. Albert Fussmann, Hans-Jürgen Palme u. Annette Sunderer. Nürnberg: Emwe-Verlag. S. 11-23.

Gaffer, Yvonne (2001). Aktionismus in der Adoleszenz. Theoretische und empirische Analysen am Beispiel von Breakdance Gruppen. Berlin: Logos.

Gebauer, Gunter; Wulf, Christoph (1998): Mimesis. Kultur - Kunst - Gesellschaft: Reinbek. Rowohlt.

Glotz, Peter (1999). Die beschleunigte Gesellschaft. Kulturkämpfe im digitalen Kapitalismus. München: Kindler.

Glotz, Peter (2004): «Rückblick auf das 21. Jahrhundert. Entwurf für ein Schweizer Geschichtsbuch Auflage 2080». Renaissance der Utopie. Zukunftsfiguren des 21. Jahrhundert. Hrsg. von Rudolf Maresch u. Florian Rötzer. Frankfurt/M.: Suhrkamp. S. 21-33.

Grob, Urs; Maag Merki, Katharina (2001). Überfachliche Kompetenzen: Theoretische Grundlegung und empirische Erprobung eines Indikatorensystems. Bern: Peter Lang. 
Groeben, Norbert (2002). «Dimensionen der Medienkompetenz: Deskriptive und normative Aspekte». Medienkompetenz: Voraussetzungen, Dimensionen, Funktionen. Hrsg. v. Norbert Groeben u. Bettina Hurrelmann. Weinheim, München: Juventa. S. 160-197.

Grundmann, Matthias (2006). Sozialisation. Skizze einer allgemeinen Theorie. Konstanz: UVK.

Herzig, Bardo (2001). "Medienerziehung und informatische Bildung. Ein (semiotischer) Beitrag zu einer integrativen Medienbildungstheorie». Medien machen Schule: Grundlagen, Konzepte und Erfahrungen zur Medienbildung. Hrsg. v. Bardo Herzig. Bad Heilbrunn/ Obb.: Klinkhardt. S. 129-164.

Höhne, Thomas (2003). Pädagogik der Wissensgesellschaft. Bielefeld: transcript.

Höhne, Thomas (2007). «Der Leitbegriff 〈Kompetenz〉 als Mantra neoliberaler Bildungsreformer. Zur Kritik seiner semantischen Weitläufigkeit und inhaltlichen Kurzatmigkeit». Bildung - Wissen - Kompetenz. Hrsg. v. Ludwig A. Pongratz, Roland Reichenbach u. Michael Wimmer. Bielefeld: Janus, S. 9-29.

Hörning, Karl Heinz (2001): Experten des Alltags. Die Wiederentdeckung des praktischen Wissens. Weilerswist: Velbrück Wissenschaft.

Hörster, Reinhard; Müller, Burkhard K. (1996). «Zur Struktur sozialpädagogischer Kompetenz. Oder: Wo bleibt das Pädagogische in der Sozialpädagogik?» In: Combe, Arno; Helsper, Werner (Hrsg.): Pädagogische Professionalität. Untersuchungen zum Typus pädagogischen Handelns. Hrsg. v. Arno Combe und Werner Helsper. Frankfurt/M.: Suhrkamp. S. 614-648.

Hubig, Christoph (Hrsg.) (2000). Unterwegs zur Wissensgesellschaft. Grundlagen - Trends Probleme. Berlin: Sigma.

Hummrich, Merle et al. (2006). «Individuation in pädagogischen Generationenbeziehungen. Passungsverhältnisse zwischen naturwüchsiger Eltern-Kind-Beziehung und pädagogischem Arbeitsbündnis». Zeitschrift für qualitative Bildungs-, Beratungs- und Sozialforschung. 7. Jg., Heft 1/2006. S. 25-46.

Immerfall, Stefan (1998). "Gesellschaftsmodelle und Gesellschaftsanalyse». Handwörterbuch zur Gesellschaft Deutschlands. 2. Aufl. Hrsg. v. Bernhard Schäfers u. Wolfgang Zapf. Opladen: Leske + Budrich. S. 253-63.

Josczok, Detlef (2001): «Bildung - kein Megathema. Ein Zwischenruf». Aus Politik und Zeitgeschichte. Beilage zur Wochenzeitung «das Parlament», 08/2001, Bd. 36. S. 33-38.

Joas, Hans; Knöbl, Wolfgang (2004). Sozialtheorie. Zwanzig einführende Vorlesungen. Frankfurt/M.: Suhrkamp.

Kerres, Michael; de Witt, Claudia; Schweer, Claudia (2003). «Die Rolle von Medienpädagoglnnen bei der Gestaltung der Medien- und Wissensgesellschaft». Beruf Medienpädagoge. Selbstverständnis - Ausbildung - Arbeitsfelder. Hrsg. von Norbert Neuss. München: kopaed. S. 87-97.

Kilius, Nelson; Kluge, Jürgen; Reisch, Linda (Hrsg.) (2002). Die Zukunft der Bildung. Frankfurt/M.: Suhrkamp.

Klafki, Wolfgang (1996). «Kann Erziehungswissenschaft zur Begründung pädagogischer Zielsetzungen beitragen?»Pädagogik und Ethik. Hrsg. v. Detlef Horster u. Kurt Beutler. Stuttgart: Reclam. S. 152-163.

Kluge, Jürgen (2003). Schluss mit der Bildungsmisere. Ein Sanierungskonzept. Frankfurt/M., New York: Campus.

Krotz, Friedrich (2007): Mediatisierung. Fallstudien zum Wandel von Kommunikation. Wiesbaden: VS Verlag. 
Kübler, Hans-Dieter (2005). Mythos Wissensgesellschaft. Gesellschaftlicher Wandel zwischen Information, Medien und Wissen. Eine Einführung. Wiesbaden: VS-Verlag.

Löffelholz, Martin; Altmeppen, Klaus-Dieter (1994). «Kommunikation in der Informationsgesellschaft». Die Wirklichkeit der Medien. Eine Einführung in die Kommunikationswissenschaft. Hrsg. v. Klaus Merten u. Siegfried J. Schmidt. Opladen: Westdeutscher Verlag. S. 570-591.

Maasen, Sabine (1999). Wissenssoziologie. Bielefeld: transcript.

Marotzki, Winfried (2004). «Von der Medienkompetenz zur Medienbildung». Lebensbegleitendes Lernen als Kompetenzentwicklung. Analysen - Konzeptionen - Handlungsfelder. Hrsg. v. Rainer Brödel u. Julia Kreimeyer. Bielefeld: W. Bertelsmann Verlag. S. 63-74.

Marotzki, Winfried; Nohl, Arnd Michael (2004). «Bildungstheoretische Dimensionen des Cyberspace». Soziologie des Cyberspace. Medien, Strukturen und Semantiken. Hrsg. v. Udo Thiedeke. Wiesbaden: VS-Verlag. S. 335-354.

May, Michael (2004): «Aneignung und menschliche Verwirklichung». "Aneignung» als Bildungskonzept der Sozialpädagogik. Beiträge zur Pädagogik des Kindes- und Jugendalters in Zeiten entgrenzter Lernzonen. Hrsg. von Ulrich Deinet u. Christian Reutlinger. Wiesbaden: VS Verlag. S. 49-70.

Messner, Rudolf (2003). «PISA und Allgemeinbildung». Zeitschrift für Pädagogik. 49. Jg., Heft 3/2003. S. 400-412.

Niesyto, Horst (2004). Medienpädagogik und soziokulturelle Unterschiede. Langfassung einer Studie auf der Basis von Experten-Interviews in Baden-Württemberg und RheinlandPfalz. Ludwigsburg: Verlag Pädagogische Hochschule Ludwigsburg.

Nohl, Arnd-Michael (2001). Migration und Differenzerfahrung. Junge Einheimische und Migranten im rekonstruktiven Milieuvergleich. Opladen: Leske + Budrich.

Nohl, Arnd-Michael (2006). Bildung und Spontaneität. Phasen biographischer Wandlungsprozesse in drei Lebensaltern - Empirische Rekonstruktionen und pragmatische Reflexionen. Opladen: Barbara Budrich.

OECD (2005). Bildung auf einen Blick. OECD-Indikatoren 2005. Heidelberg: Spektrum Akademischer Verlag.

Otto, Peter; Sonntag, Phillip (1985). Wege in die Informationsgesellschaft. Steuerungsprobleme in Wirtschaft und Politik. München: DTV.

Paus-Hasebrink, Ingrid (2005). Grundsätzliche Überlegungen zu «neuen Denkwerkzeugen» in der Jugend(medien)forschung. In: Kleber, Hubert (Hrsg.): Perspektiven der Medienpädagogik in Wissenschaft und Bildungspraxis. München. kopaed. S. 74-85.

Pfeiffer, Christian; Mössle, Thomas ; Kleimann, Matthias; Rehbein, Florian (2007): Die PISA-Verlierer - Opfer ihres Medienkonsums. Eine Analyse auf der Basis verschiedener empirischer Untersuchungen. http://www.kfn.de/versions/kfn/assets/pisaverlierer.pdf (26.01.2008).

Pietrass, Manuela (2002). «Medienbildung». Handbuch Bildungsforschung. Hrsg. v. Rudolf Tippelt. Opladen: Leske + Budrich. S. 393-408.

Pietrass, Manuela (2005). «Für alle alles Wissen jederzeit... Grundlagen von Bildung in der Mediengesellschaft». Perspektiven der Medienpädagogik in Wissenschaft und Bildungspraxis. Hrsg. v. Hubert Kleber. München: kopaed. S. 39-50.

Radtke, Frank-Olaf (2006). «Erziehung, Markt und Gerechtigkeit». Zeitschrift für Pädagogik 52. Jg., Heft 1, Januar/Februar 2006. S. 52-59.

Rammert, Werner (2000). Technik aus soziologischer Perspektive; Band 2: Kultur - Innovation - Virtualität. Opladen: Westdeutscher Verlag. 
Ruhloff, Jörg (2006). «Die Universität ist kein Wirtschaftsbetrieb». Unternehmen Bildung. Die Frankfurter Einsprüche und kontroverse Positionen zur aktuellen Bildungsreform. Sonderheft zur Vierteljahresschrift für wissenschaftliche Pädagogik. Hrsg. v. Ursula Frost. Paderborn: Ferdinand Schöningh. S. 25-30.

Schäffer, Burkhard (1996). Die Band - Stil und ästhetische Praxis im Jugendalter. Opladen: Leske + Budrich.

Schell, Fred (1999). «Bedeutung von Medienkompetenz als Bildungsaufgabe und inhaltliche bildungspolitische Zielsetzung». Medienkompetenz. Grundlagen und pädagogisches Handeln. Hrsg. v. Fred Schell, Elke Stolzenburg u. Helga Theunert. München: kopaed. S. 272-281.

Scherr, Albert (2002). «Der Bildungsauftrag der Jugendarbeit: Aufgaben und Selbstverständnis im Spannungsfeld von sozialpolitischer Indienstnahme und aktueller Bildungsdebatte». Bildung und Lebenskompetenz. Kinder- und Jugendhilfe vor neuen Aufgaben. Hrsg. v. Richard Münchmeier, Hans-Uwe Otto u. Ursula Rabe-Kleberg. Opladen: Leske + Budrich. S. 93-106.

Stehr, Nico (1994): Arbeit, Eigentum und Wissen. Zur Theorie von Wissensgesellschaften. Frankfurt/M.: Suhrkamp.

Stehr, Nico (2001). «Moderne Wissensgesellschaften». Aus Politik und Zeitgeschichte. Beilage zur Wochenzeitung «das Parlament», 08/2001, Bd. 36. S. 7-14.

Steinbicker, Jochen (2001): Zur Theorie der Informationsgesellschaft. Opladen: Leske + Budrich.

Stross, Annette M. (2001). « «Wissensgesellschaft» und Reformpädagogik im aktuellen bildungspolitischen Diskurs oder: Über notwendige Synthetisierungsleistungen der Erziehungswissenschaft». Der pädagogische Blick. Zeitschrift für Wissenschaft und Praxis in pädagogischen Berufen 9, 2001, Heft 4. S. 207-216.

Sutter, Tilmann; Charlton, Michael (2002). «Medienkompetenz - einige Anmerkungen zum Kompetenzbegriff». Medienkompetenz: Voraussetzungen, Dimensionen, Funktionen. Hrsg. v. Norbert Groeben u. Bettina Hurrelmann. Weinheim, München: Juventa. S. 129147.

Tenorth, Heinz-Elmar (1997). «Bildung» - Thematisierungsformen und Bedeutung in der Erziehungswissenschaft». Zeitschrift für Pädagogik, 43. Jg. 1997, Nr. 6. S. 969-984.

Thole, Werner (2003): «Reflexivität und Eigensinn in einem diffusen Feld. Bildung nach PISA und die Kinder- und Jugendarbeit - vorsichtige Hinweise auf verhüllte oder vergessene Zusammenhänge». Kinder- und Jugendarbeit als Bildungsprojekt. Hrsg. von Werner Lindner, Werner Thole u. Jochen Weber. Opladen: Leske + Budrich. S. 247-262.

Voss, Günter G. (2000). «Alltag: Annäherungen an eine diffuse Kategorie». Neue Medien im Alltag. Begriffsbestimmungen eines interdisziplinären Forschungsfeldes. Hrsg. v. Günter G. Voss, Werner Holly u. Klaus Boehnke. Opladen: Leske + Budrich. S. 31-77.

Watt, D. H. (1980). "Computer literacy: what should schools be doing about this?» Classroom Computer News, 1 (2). S. 1-26.

Weber, Peter J. (2002). "Technisierung und Marktorientierung von Bildung in Europa». Die verkaufte Bildung. Kritik und Kontroversen zur Kommerzialisierung von Schule, Weiterbildung, Erziehung und Wissenschaft. Hrsg. v. Ingrid Lohmann u. Rainer Rilling Opladen: Leske + Budrich. S. 29-43.

Welling, Stefan (2008): Computerpraxis Jugendlicher und medienpädagogisches Handeln. München. kopaed. 
Wimmer, Michael (2002). «Bildungsruinen in der Wissensgesellschaft. Anmerkungen zum Diskurs über die Zukunft der Bildung». Die verkaufte Bildung. Kritik und Kontroversen zur Kommerzialisierung von Schule, Weiterbildung, Erziehung und Wissenschaft. Hrsg. v. Ingrid Lohmann u. Rainer Rilling Opladen: Leske + Budrich. S. 45-68.

Zacharias, Wolfgang (2004). «Zum Beispiel AG Inter@ktiv in München. Kommunale Netzwerk-Infrastrukturen - zwischen 〈Lebenswelt \& Internet) und zwischen «Sinn \& Cyber»». Informelle Bildung online. Perspektiven für Bildung, Jugendarbeit und Medienpädagogik. Hrsg. v. Hans-Uwe Otto u. Nadia Kutscher. München: Weinheim: Juventa. S. 167187.

Zirfas, Jörg; Wulf, Christoph (2001). «Integration im Ritual. Performative Prozesse und kulturelle Differenz». Zeitschrift für Erziehungswissenschaft 4, Heft 2. S. 191-208. 